\title{
Desafios enfrentados no processo de inclusão de indivíduos com transtorno do espectro autista no âmbito universitário
}

\author{
Challenges faced in the process of inclusion of individuals with autistic spectrum disorder at the \\ university scope \\ Desafíos enfrentados en el proceso de inclusión de personas con trastorno del espectro autista en el \\ ámbito universitário
}

Recebido: 23/03/2021 | Revisado: 29/03/2021 | Aceito: 01/04/2021 | Publicado: 12/04/2021

\author{
Beatriz de Brito Pinto \\ ORCID: https://orcid.org/0000-0002-5972-763X \\ Centro Universitário Católico de Quixadá, Brasil \\ E-mail: biapintob@hotmail.com \\ Maria Caroline Ribeiro Maciel \\ ORCID: https://orcid.org/0000-0002-9879-8350 \\ Universidade Federal do Espírito Santo, Brasil \\ E-mail: maria.c.maciel@edu.ufes.br \\ Priscilla Oliveira da Silva \\ ORCID: https://orcid.org/0000-0003-3003-7368 \\ Universidade do Estado do Amazonas, Brasil \\ E-mail: pos.odo18@uea.edu.br \\ Milena Roberta Freire da Silva \\ ORCID: https://orcid.org/0000-0003-0203-4506 \\ Universidade Federal de Pernambuco, Brasil \\ E-mail: milena.freire@ufpe.br
}

\begin{abstract}
Resumo
Introdução: O Transtorno do Espectro Autista (TEA) designa inúmeras manifestações relacionadas às deficiências na comunicação e interação social. Em virtude da grande inserção desses indivíduos no âmbito universitário, faz-se necessário compreender como esse processo ocorre, assim como quais desafios são vivenciados. Objetivo: Discorrer e elencar os principais desafios no processo de inclusão enfrentados por indivíduos com TEA no âmbito da educação de ensino superior. Métodos: Trata-se de uma revisão integrativa, onde foram realizadas buscas nas bases eletrônicas Scielo, Lilacs, BVS e MedLine, utilizando os descritores de acordo com o DeSC, publicados entre 2017 e 2021 . Para compor os resultados foram selecionados sete artigos de acordo com os critérios de inclusão e exclusão previamente estabelecidos. Resultados: Os principais desafios enfrentados por indivíduos com TEA no processo de inclusão são ansiedade, depressão, medo, falta de compreensão por parte do corpo docente, dificuldades em adaptações as metodologias de ensino, dificuldade de socialização, assim como estigma e falta de suporte individualizado, não sendo respeitada as singularidades de cada indivíduo. Conclusão: Mesmo com o crescente número de estudantes com TEA nas universidades, ainda são evidentes as dificuldades enfrentadas, assim como as limitações apresentadas pelas IES para lidarem com esse público, por isso faz-se necessário a adoção de medidas que sejam capazes de priorizar esses indivíduos.
\end{abstract}

Palavras-chave: Inclusão; Ensino superior; Autismo.

\begin{abstract}
Introduction: Autistic Spectrum Disorder (ASD) designates numerous manifestations related to deficiencies in communication and social interaction. Due to the great insertion of these individuals in the university environment, it is necessary to understand how this process occurs, as well as what challenges are experienced. Objective: To discuss and list the main challenges in the inclusion process faced by individuals with ASD in the context of higher education. Methods: This is an integrative review, where searches were carried out in the electronic databases Scielo, Lilacs, VHL and MedLine, using the descriptors according to the DeSC, published between 2017 and 2021. To compose the results, seven articles were selected according to with the inclusion and exclusion criteria previously established. Results: The main challenges faced by individuals with ASD in the inclusion process are anxiety, depression, fear, lack of understanding by the faculty, difficulties in adapting teaching methodologies, difficulty in socialization, as well as stigma and lack of individualized support., not being respected the singularities of each individual. Conclusion: Even with the growing number of students with ASD in universities, the difficulties faced are still evident, as well as the limitations presented by HEIs to deal with this audience, so it is necessary to adopt measures that are able to prioritize these individuals.
\end{abstract}


Keywords: Inclusion; University education; Autism.

\section{Resumen}

Introducción: El trastorno del espectro autista (TEA) designa numerosas manifestaciones relacionadas con deficiencias en la comunicación y la interacción social. Debido a la gran inserción de estos individuos en el ámbito universitario, es necesario comprender cómo se da este proceso, así como qué desafíos se viven. Objetivo: Discutir y enumerar los principales desafíos en el proceso de inclusión que enfrentan las personas con TEA en el contexto de la educación superior. Métodos: Se trata de una revisión integradora, donde se realizaron búsquedas en las bases de datos electrónicas Scielo, Lilacs, BVS y MedLine, utilizando los descriptores según el DeSC, publicados entre 2017 y 2021. Para componer los resultados se seleccionaron siete artículos según con los criterios de inclusión y exclusión previamente establecidos. Resultados: Los principales desafíos que enfrentan las personas con TEA en el proceso de inclusión son ansiedad, depresión, miedo, incomprensión por parte del profesorado, dificultades para adaptar las metodologías de enseñanza, dificultad en la socialización, así como el estigma y la falta de apoyo individualizado., No siendo respetadas las singularidades de cada individuo. Conclusión: Aún con el creciente número de estudiantes con TEA en las universidades, las dificultades que enfrentan aún son evidentes, así como las limitaciones que presentan las IES para atender a esta audiencia, por lo que es necesario adoptar medidas que sean capaces de priorizar a estos individuos.

Palabras clave: Inclusión; Enseñanza superior; Autismo.

\section{Introduçãa}

O Transtorno do Espectro Autista (TEA), designa uma pluralidade de manifestações relacionadas as deficiências na comunicação e interação social, no entanto, podem apresentar características mais específicas, como problemas de coordenação motora e de equilíbrio, dificuldade para iniciar movimentos, alterações sensoriais (auditivas, visuais, olfativas, táteis e gustativas), percepção a dor diminuída, alteração de linguagem, jogo simbólico empobrecido e problemas na alimentação, podendo estar associado a convulsões e a outras deficiências, essas características apresentam-se em diferentes níveis de severidade nas pessoas com esse transtorno (Benitez et al., 2017; Ramos; Lemos; Salomão, 2019; Silveira; Donida; Santana, 2020).

A abordagem do TEA no âmbito escolar ocorre desde 1994 por meio da Política Nacional de Educação Especial. No Brasil, o processo de inclusão escolar ocorre de maneira variada, mesmo com a promulgação dos documentos normativos que regem estratégias acerca dessa temática. No ano de 2012 foi instaurada a Lei 12.764/ 2012 que institui a Política Nacional de Proteção aos Direitos da Pessoa com Transtorno do Espectro Autista. Essa lei tem como princípio fornecer orientações para a educação das pessoas com TEA, garantindo assim o processo de escolarização, sejam em escolas comuns ou de atendimento educacional especializado (AEE), objetiva também fortalecer as ações de políticas públicas e saúde voltadas para esse público, bem como estimular o acesso ao mercado de trabalho, à pesquisa científica, a capacitações e a formações profissionais (Wuo, 2019)

Resultado de um processo histórico de luta contra o capacitismo atrelado às deficiências, o ingresso de estudantes com TEA tem aumentado significativamente na educação superior. Neste cenário, aspectos legais têm colaborado para a acessibilidade e ingresso nas instituições de ensino superior (IES), a exemplo das bolsas de estudo ofertadas através do Programa Universidade para Todos (ProUni) destinadas a deficientes em faculdades privadas e o Programa de Acessibilidade na Educação Superior - Incluir - para universidades públicas (Silva et al. 2020). Dessa forma, as políticas públicas brasileiras têm contribuído paulatinamente para o processo de inclusão à medida que auxiliam as instituições no cumprimento de sua responsabilidade de adequar-se para atender as singularidades existentes do sujeito, rompendo com paradigmas préestabelecidos da academia em que os desvios do considerado "padrão" não são aceitos (Almeida \& Ferreira, 2018)

Contudo, evidencia-se que, apesar dessas políticas públicas auxiliarem efetivamente no ingresso ao ensino superior, as ações direcionadas para a permanência ainda apresentam empecilhos para implementação (Almeida \& Ferreira, 2018). Nesta ótica, faz-se necessário adaptações curriculares na educação superior para atender as dificuldades de aprendizagem dos 
estudantes, e assim diminuir as barreiras de inclusão desse público-alvo de educação especial. Esse processo é fundamental pois possibilita uma educação democrática, que seja para todos e que garanta a permanência e uma trajetória acadêmica de qualidade. Todavia, as especificidades desses estudantes ainda não são totalmente contempladas, uma vez que tanto a escola como a universidade direcionam para práticas pedagógicas excludentes (Silva \& Pavão, 2018). Nesta perspectiva de inclusão de indivíduos com TEA, o presente estudo tem como objetivo discorrer e elencar os principais desafios no processo de inclusão enfrentados por estudantes com TEA no âmbito da educação do ensino superior.

\section{Métodos}

Para a elaboração da presente revisão integrativa, os seguintes passos foram seguidos: identificação do tema e seleção da questão de pesquisa, estabelecimento de critérios para inclusão e exclusão de estudos, definição das informações a serem extraídas dos estudos selecionados, avaliação dos estudos incluídos na revisão integrativa, interpretação dos resultados e apresentação da revisão (Galvão, 2008; Souza et al. 2010).

Inicialmente, foi elaborada a questão norteadora: "Quais os desafios enfrentados no processo de inclusão para indivíduos com transtorno do espectro autista no âmbito universitário?". Subsequente foram elencados os critérios para seleção dos artigos, sendo os de inclusão: textos completos compatíveis com o assunto de interesse, artigos indexados em português, inglês ou espanhol e publicados entre 2017 e 2021. Os critérios de exclusão foram: artigos duplicados, artigos que não atendem a temática, editoriais, teses e dissertações.

A seleção dos estudos foi realizada a partir de buscas em quatro bases de dados: Scielo (Scientific Electronic Library Online), BVS (Biblioteca Virtual em Saúde), Lilacs (Literatura Latino-Americana e do Caribe em Ciências da Saúde) e MedLine (Sistema Online de Busca e Análise de Literatura Médica). Para o direcionamento da pesquisa foram selecionados os descritores através do DeCS, sendo esses: Educação especial (Education, Special), Educação de Pessoa com Deficiência Intelectual (Education of Intellectually Disabled), Inclusão Escolar (Mainstreaming, Education) e Transtorno do espectro Autista (Autism Spectrum Disorder), usando o operador booleano "and".

Como resultado das buscas nas bases de dados supracitadas, foram encontrados 177 artigos, utilizando os descritores e os critérios de inclusão estabelecidos. Após análise minuciosa, para compor os resultados foram selecionados apenas sete, uma vez que, a partir da leitura dos estudos na íntegra apenas esses se enquadram na proposta do estudo (Figura 1).

No tocante da classificação dos estudos quanto aos níveis de "evidencia", foi utilizada a categorização estabelecida por Melnyk; Fineout-Overholt (2011). São organizados por um sistema de classificação que divide os estudos em sete níveis: nível I - evidências provenientes de revisões sistemáticas, meta análises, ensaios clínicos randomizados controlados; nível II evidências obtidas de ensaios clínicos randomizados bem delineado; nível III - evidências originadas de ensaios clínicos bem delineados sem randomização; nível IV - evidências derivadas de estudos do tipo caso controle e coorte; nível V- evidências procedentes de revisões sistemáticas de estudos descritivos e qualitativos; nível VI - evidências retiradas de um único estudo qualitativo ou descritivo; e nível VII - evidências propostas pela opinião de autoridades e/ou relatórios emitidos por comitês de especialistas. 
Figura 1. Fluxograma de seleção dos artigos sobre os desafios na inclusão de indivíduos com TEA no âmbito universitário.
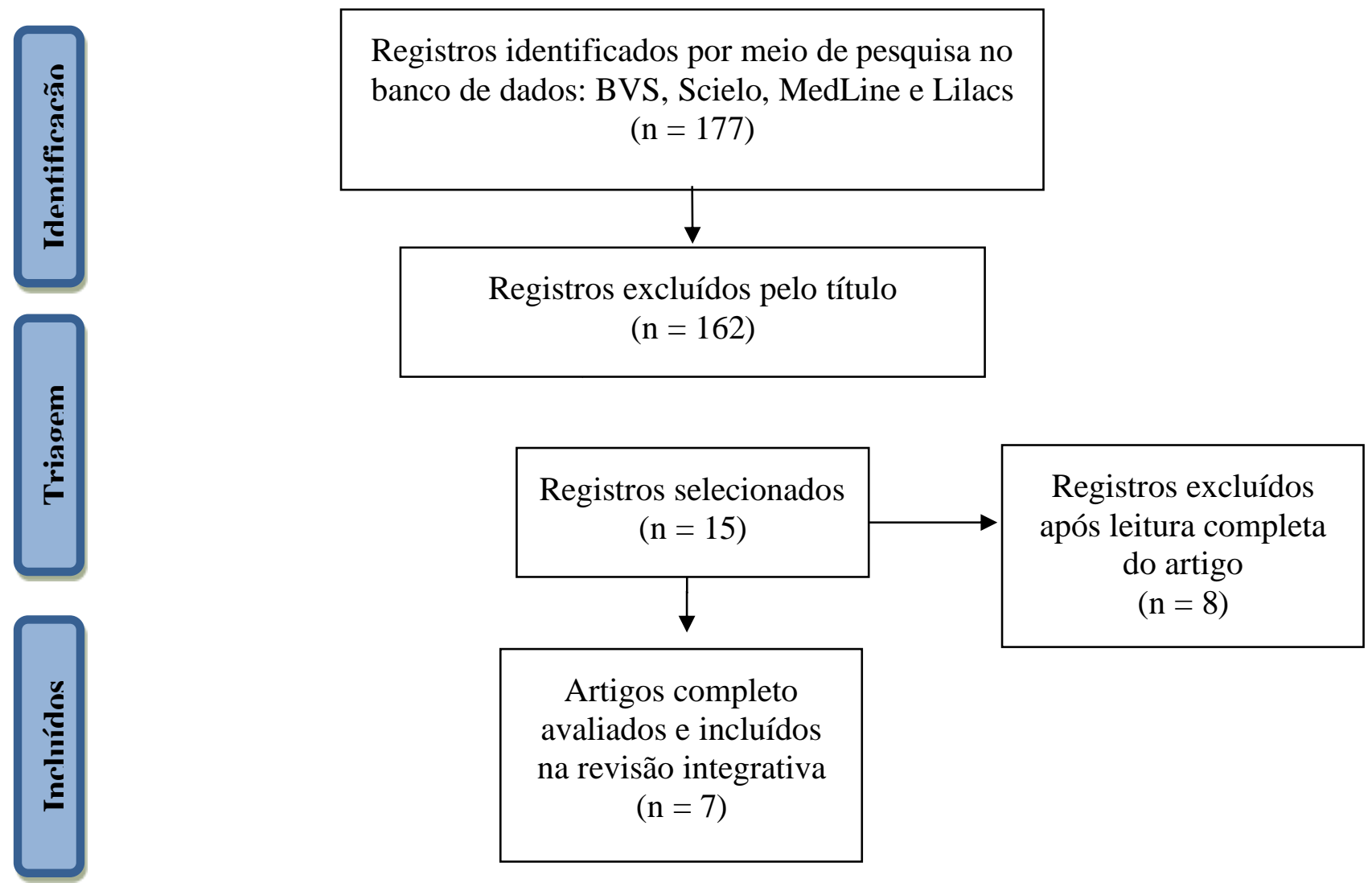

Fonte: Autores (2021).

\section{Resultados e Discussão}

Nesta revisão integrativa foram selecionados sete artigos de acordo com os critérios de inclusão pré-definidos para posterior análise. Dentre os artigos incluídos, cinco encontravam-se em língua inglesa (71,42\%) e dois (28,57\%) em língua portuguesa, sendo dois com publicação datada em 2017 (28,57\%), um em 2018 (14,28\%), dois em 2019 (28,57\%) e dois em 2020 (28,57\%). Em relação às bases de dados nas quais foram identificados, um (14,28\%) foi identificado na Scielo, um $(14,28 \%)$ na Medline, três $(42,85 \%)$ na BVS e dois $(28,57 \%)$ na Lilacs. A fim de sintetizar os resultados encontrados nos artigos, tais como título, autor, ano, tipo e nível de evidência do estudo, objetivo do estudo, local e, sobretudo, os principais achados em relação à problemática tratada nesta revisão, foi elaborado um quadro-síntese (Tabela 1). 
Tabela 1. Descrição dos estudos referente a inclusão dos estudantes com TEA no âmbito universitário.

\section{Título}

Objetivo

\section{Tipo de}

estudo/Nível

evidência

\section{Local de estudo}

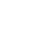 \\ Des:}

Desa

Autor/Ano
An On-Line Survey of University Students with Autism Spectrum Disorder in Australia and New Weográfico na Austrália e Nova cart cacterísticas dos alunos com TEA

Realizar um levantamento de cunho $\begin{array}{cc}\text { Estudo quantitativo } & \text { Austrália e Nova } \\ \mathrm{V} & \text { Zelândia }\end{array}$ Zelândia

- Depressão;

- Problemas de saúde mental;

- Qualidade do sono;

- Sensibilidades sensoriais;

- Requisitos Acadêmico;

- Requisitos sociais na universidade.

Identificar, do ponto de vista do estudante com Transtorno do Desafios da inclusão: a invisibilidade Espectro Autista (TEA) o processo das pessoas com Transtorno do de adaptaça a enfrentadas, as estratégias utilizadas e os recursos estrategias utilizadas e os recuridos pelas universidades
oferis
Estudo qualitativo, do tipo exploratório descritiva VI

- Estigma;

- Desconhecimento do TEA por parte de professores e colegas;

- Características próprias do autismo como disfunção executiva;

- Ansiedade;

- Alterações sensoriais; F., 2020 .

Anderson, A. H.; Carter M.; Stephenson J., 2020.

- Dificuldade no relacionamento com colegas;

- Dificuldades em adaptações didáticopedagógicas;

- Dificuldades no relacionamento com os profissionais do núcleo de apoio.

Analisar o processo de inserção de College Access, Success and Equity alunos com TEA na universidade for Students on the Autism Spectrum por meio da investigação de questões como o acesso, sucesso e equidade.
- Falta de compreensão por parte dos tutores;

- Assédio moral;

- Falta de suporte individualizado;

Estudo quantitativo Estados Unidos da

V América

- Professores inflexíveis;

- Depressão;

- Ansiedade;

- Baixa auto estima;

- Medos.
Accardo, A. L. et al. 2019. 
Experiências Acadêmicas de Descrever experiência acadêmica de Estudantes Universitários com universitários com diagnóstico de Transtorno do Espectro Autista: uma TEA matriculados em universidade Análise Interpretativa dos Relatos
- Dificuldade dos professores em compreender as singularidades do estudante com TEA;

- Déficits de concentração nas aulas;

Olivati, A.G.; Leite, L.P.,
Estudo quantitativo $\mathrm{V}$

Brasil pública de São Paulo.
- Ausência de preparação de alunos e professores:

- Falta de serviços e auxílios.

- Dificuldade de interação.
Autism and the University Compreender as perspectivas pósExperience: Narratives from $\begin{aligned} & \text { Compreender as perspectivas pós- } \\ & \text { secundárias de alunos com }\end{aligned}$ ith Neurodevelopmental Disorders $\begin{aligned} & \text { secundárias de alunos com } \\ & \text { distúrbios }\end{aligned}$
de

neurodesenvolvimento

\section{Estudo quantitativo} V
Estados Unidos da América
- Demandas acadêmicas estressantes;

- Pressão por parte do corpo docente;

- Dificuldade de socialização entre os alunos.
Bolourian, Y.; Zeedyk, S.

M.; Blacher, J., 2018.

- Estresse devido à quebra de rotina na transição do ensino médio ao ensino superior;

Young Adults on the Autism Compreender questões específicas Spectrum at College: Successes and relacionadas ao sucesso ou fracasso de pessoas com TEA no ensino Stumbling Block superior.

\section{Estudo qualitativo Estados Unidos d} América

- Ansiedade;

- Dificuldade na adesão de disciplinas obrigatórias que não os interessam;

- Estigma.

Reunir informações acerca de experiências acadêmicas, sociais e de habitação no âmbito universitário Autism and Accommodations in a partir da perspectiva de pessoas Higher Education: Insights from the com Autism Community possíveis mudanças em prol da melhoria no que diz respeito a inclusão dessas pessoas no ensino superior. $\begin{array}{cc}\text { Estudo qualitativo } & \text { Estados Unidos da } \\ \text { VI } & \text { América }\end{array}$

- Insatisfação com as adaptações acadêmicas voltadas para a inclusão;

- Dificuldade de adesão dos discentes as adaptações acadêmicas;

- Exclusão das oportunidades de bolsas de estudos;

- Dificuldade para interagir e se comunicar com alunos neurotípicos;

- Mediadores e mentores sem preparo para lidar com a neurodiversidade;

- Falta de um espaço favorável ao autismo no campus.

no

Fonte: Autores (2021). 
A partir da análise dos estudos supracitados foi possível realizar uma categorização através da construção de tópicos que abordem de forma detalhada o objetivo proposto pelo presente artigo, além de possibilitar uma melhor comparação entre os resultados demonstrados.

\section{Desafios psicossociais enfrentados por universitários com TEA}

Os principais desafios enfrentados no processo de inclusão por esses indivíduos com TEA são a ansiedade, depressão, baixo auto estima, medos, estigmas sociais (Anderson; Carter; Stephenson, 2020; Aguilar \& Rauli, 2020; Accardo et al., 2019), além de dificuldades no relacionamento com os profissionais do núcleo de apoio, assim como entre os próprios alunos da instituição de ensino (Figura 2) (Bolourian; Zeedyk; Blacher, 2018).

Figura 2 - Desafios psicossociais vivenciados por universitários com TEA.

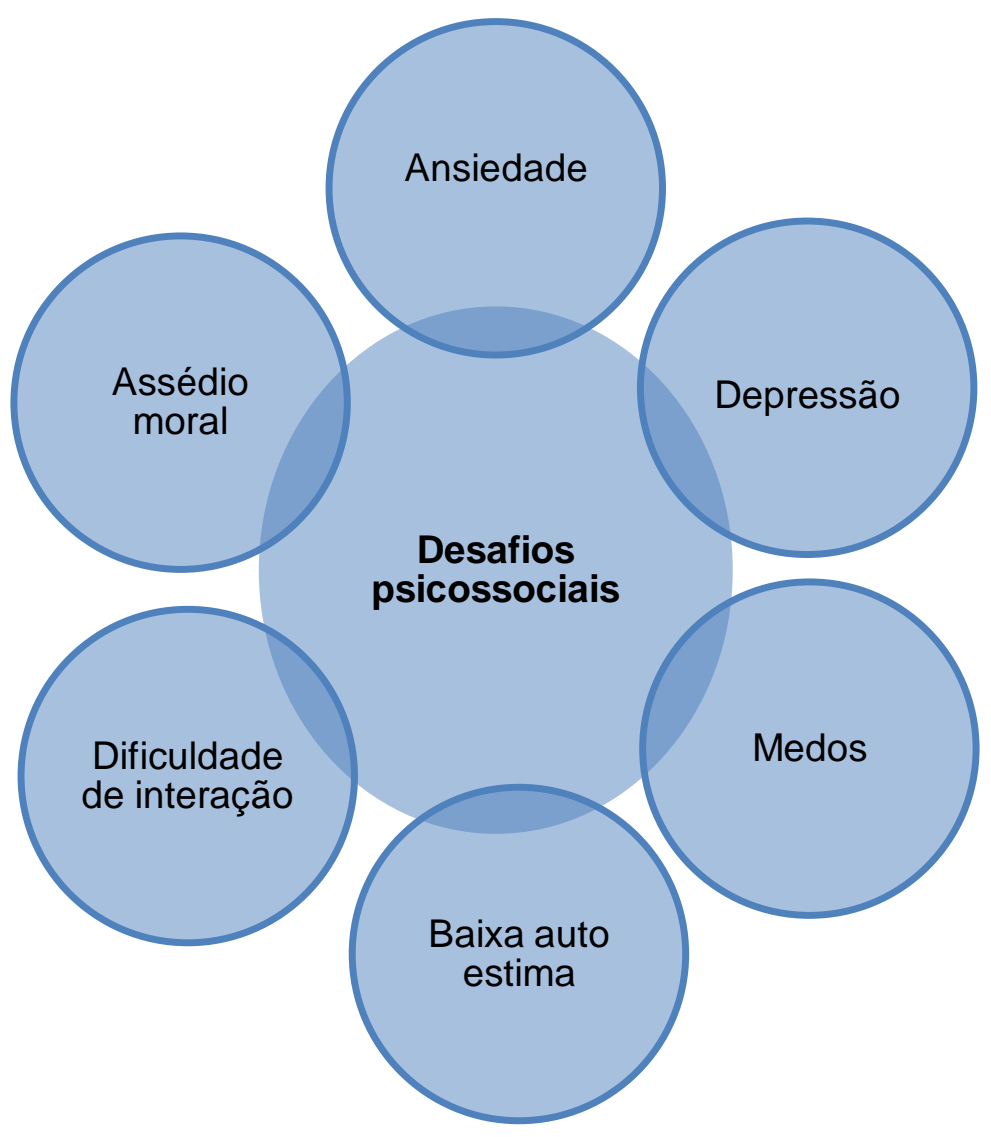

Fonte: Autores (2021).

Um fator relevante apresentado por Assis et al. (2021), é o aumento na incidência do sofrimento mental durante o período universitário. Isto se deve ao fato de que, ao chegarem à universidade, esses estudantes se deparam com um ambiente que exige deles mais autonomia e organização. Esta nova estrutura pode gerar fatores estressores e ansiosos, pois a nova rotina ainda não foi preestabelecida, implicando no seu desempenho acadêmico. Vale ressaltar que nos discentes com TEA, a probabilidade de desenvolver condições psiquiátricas duplica. Além disso, estes podem ter concomitante ao TEA sofrimentos psíquicos pré-existentes (Gonçalves, 2020).

Anderson e Butt (2017) enfatizam que a desatenção voltada às habilidades interativas dos alunos com TEA em detrimento do seu sucesso acadêmico escolar leva a um não preparo dos mesmos perante o enfrentamento dos obstáculos de 
caráter social presentes no ensino superior. Dessa forma, os adventos do fim do ensino médio e a subsequente entrada para a universidade tornam-se desencadeadores de estresse, medo, ansiedade e até mesmo violência e ataques de pânico.

No tocante aos obstáculos sociais enfrentados pelos estudantes com TEA, está presente o estigma (Anderson \& Butt, 2017; Aguilar \& Rauli, 2020). Indivíduos com TEA são comumente estigmatizados devido a associação desse transtorno a incapacidades sociais e intelectuais e comportamentos aos quais geram "estranhamento" (Fernandes, 2020). É importante salientar que, como relatado no estudo realizado por Cox et al. (2017), a partir desse processo de estigmatização, surgem outras problemáticas, como o preconceito e a dificuldade das pessoas com TEA de aceitarem suas próprias características. Assim sendo, o estigma imposto sobre esses estudantes corrobora para uma maior dificuldade de socialização e a consequente segregação desses indivíduos.

\section{Desafios referentes ao apoio promovido pelas universidades}

A inserção de pessoas com TEA em universidades aumentou no decorrer dos anos devido ao apoio prestado aos estudantes e a iniciativas legais que auxiliam o ingresso do público-alvo da educação especial, além do aumento da conscientização, reconhecimento e diagnósticos de TEA. (Assis et al., 2021). No entanto, apesar desse processo de inserção estar mais avançado é notório que esses indivíduos ainda enfrentam desafios, como dificuldades em adaptações didáticopedagógicas, insatisfação com as adaptações acadêmicas voltadas para a inclusão, dificuldade dos professores em compreenderem as singularidades apresentadas pelos estudantes com TEA, além da vivencia de uma educação excludente, uma vez que não são ofertados serviços e auxílios como bolsas, exclusivamente para essas pessoas, além da ausência de preparo tanto dos professores como alunos para conviver com as limitações apresentadas por esses indivíduos (Aguilar \& Rauli,, 2020; Olivati \& Leite, 2019; Anderson \& Butt, 2017; Sarrett, 2017).

Para que a educação inclusiva seja, de fato, efetivada, faz-se necessário que as instituições educativas se comprometam com a não exclusão de estudantes devido quaisquer limitações e/ou dificuldades, assumindo a responsabilidade de educar a todos, sendo assim, corresponsáveis pelo processo de sucesso ou fracasso escolar dos estudantes (Gonçalves, 2020). Nos estudos analisados, a insatisfação em relação ao comportamento do corpo docente foi uma problemática recorrente, fato este que evidencia a falta de preparo dada a estes profissionais. Ou seja, mesmo que o aluno com TEA tenha direito à entrada e participação no ensino superior, sua permanência no ambiente acadêmico é, ainda, muito dificultosa.

A vista disso, constatou-se que a dificuldade de compreensão dos professores quanto às peculiaridades inerentes ao TEA é um importante desafio no processo inclusivo (Aguilar \& Rauli, 2020; Acarddo, et al., 2019; Olivati \& Leite, 2019; Sarrett, 2017). Nesse sentido, o estudo desenvolvido por Balbino et al. (2020), evidenciou que essa deficiência na formação dos professores acarreta impactos diretos na consolidação da inclusão escolar. Justifica-se que, a formação acadêmica não prepara esses profissionais para demandas específicas como trabalhar com pessoas com TEA.

Referente às dificuldades no relacionamento com o núcleo de apoio, é notório que os profissionais precisam ter uma formação complementar, pois o desconhecimento das bases teóricas que sustentam a proposta de inclusão dos alunos com TEA na universidade, aliado à própria inexperiência dos órgãos gestores das instituições de ensino superior, favorecem o estabelecimento de um olhar estereotipado sobre o autismo (Abreu \& Oliveira, 2019). Em vista disto, é necessário que os centros de apoio entendam as singularidades dos indivíduos portadores do TEA para poder, de fato, auxiliar no processo de inclusão destes no âmbito universitário.

Dentre as dificuldades referentes ao corpo docente apresentadas pelos estudantes com TEA, está a insatisfação em relação às adaptações didático-pedagógicas decorrente da metodologia aplicada em avaliações, que podem confundir esses alunos por apresentarem margens interpretativas diversas (Rocha et al., 2018). Ademais, outra questão relevante, conforme Assis, et al. (2021), é a evidente necessidade para que haja uma flexibilização nos prazos de entrega dos trabalhos, uma vez 
que, esses estudantes apresentam dificuldades em assimilar conteúdos novos, e, portanto, requerem mais tempo para o processo de execução. Assim sendo, evidencia-se a importância da compreensão por parte do professor acerca das individualidades do estudante para que o mesmo possa adequar sua metodologia de acordo com as demandas necessárias para que o aprendizado aconteça.

Nessa perspectiva, ressalta-se que, como posto pelos autores dos artigos analisados neste estudo, a falta de preparo dos profissionais para lidar com as singularidades das pessoas com TEA se torna uma problemática a qual requer atenção das IES, pois decorrente deste fator, são estabelecidas barreiras a efetivação do processo inclusivo no meio acadêmico (Sarrett, 2017; Bolourian \& Blacher, 2018; Accardo, et al., 2019; Olivati \& Leite, 2019; Aguilar \& Rauli, 2020).

\section{Considerações Finais}

Em face das dificuldades apresentadas pelos estudantes do ensino superior com TEA observa-se que os principais desafios enfrentados estão atrelados principalmente à falta de preparo do corpo docente para lidar com as limitações desses indivíduos assim como a uma educação excludente, uma vez que não priorizam as individualidades e limitações dos portadores de autismo. Decorrente do grande estresse no meio acadêmico é constatado o surgimento de condições psiquiátricas, como depressão, ansiedade e medo, além da dificuldade de socialização.

Fica clara a premente necessidade para que as IES, em um esforço contínuo, se comprometam com o preparo de seus profissionais para que os mesmos possam atuar de maneira verdadeiramente inclusiva, além do desenvolvimento de medidas que visem a redução desses danos psiquiátricos, para que esses alunos possam ter um melhor aproveitamento acadêmico.

Ademais, faz-se necessário que as vigentes adaptações curriculares sejam revistas, renovadas e asseguradas, de forma a contribuir com a permanência desses indivíduos no ensino superior a partir da diminuição de possíveis fatores estressores.

Finalmente, é importante relatar o papel que o estigma exerce no que tange a não valorização e respeito para com as pessoas com TEA, contribuindo, assim, com produção e propagação de fatores segregadores, dessa forma, é imprescindível a necessidade de que esses estigmas sejam quebrados não só no meio acadêmico, mas sim na sociedade como um todo.

Assim, são importantes novas abordagens na literatura científica que demonstrem o quantitativo de alunos com TEA no ensino superior por regiões brasileiras e quais as medidas didáticas pedagógicas podem ser adotadas para melhorar seu acesso e inclusão. Outro aspecto relevante é as contribuições da educação continuada para profissionais da educação sobre as peculiaridades do TEA para a efetividade do processo inclusivo.

\section{Referências}

Accardo, A. L., Bean, K., Cook, B., Gillies, A., Edgington, R., Kuder, S. J., \& Bomgardner, E. M. (2019). College access, success and equity for students on the autism spectrum. Journal of autism and developmental disorders, 49(12), 4877-4890.

Aguilar, C. P. C., \& Rauli, P. F. (2020). Desafios da inclusão: a invisibilidade das pessoas com Transtorno do Espectro Autista no ensino superior. Revista Educação Especial, 33, 43-1.

Anderson, A. H., Carter, M., \& Stephenson, J. (2020). An on-line survey of university students with autism spectrum disorder in Australia and New Zealand: Characteristics, support satisfaction, and advocacy. Journal of autism and developmental disorders, 50(2), 440-454.

Anderson, C., \& Butt, C. (2017). Young adults on the autism spectrum at college: Successes and stumbling blocks. Journal of Autism and Developmental Disorders, 47(10), 3029-3039.

Assis, L. V., Soares, G. F. G., Quixabeira, A. A., Fortes, B. C. R., Varela, C. N. C., Hubinger, G. P., \& de Souza Lima, M. F. (2021). Transtorno do Espectro Autista na percepção de acadêmicos universitários: uma revisão de literatura. Revista Eletrônica Acervo Científico, 18 , e5667-e5667.

Balbino, E. M. S., da Silva, S. G., de Oliveira, N. C. S., \& Balbino, E. S. (2021). O Aluno com Transtorno do Espectro Autista e o mediador escolar: um olhar inclusivo. Diversitas Journal, 6(1), 1593-1605.

Benitez, P., Gomes, M., Bondioli, R., \& Domeniconi, C. (2017). Mapeamento das estratégias inclusivas para estudantes com deficiência intelectual e autismo. Psicologia em Estudo, 22(1), 81-93. 
Research, Society and Development, v. 10, n. 4, e31010414189, 2021

(CC BY 4.0) | ISSN 2525-3409 | DOI: http://dx.doi.org/10.33448/rsd-v10i4.14189

Bolourian, Y., Zeedyk, S. M., \& Blacher, J. (2018). Autism and the university experience: Narratives from students with neurodevelopmental disorders. Journal of Autism and Developmental Disorders, 48(10), 3330-3343.

Cox, B. E., Thompson, K., Anderson, A., Mintz, A., Locks, T., Morgan, L., \& Wolz, A. (2017). College experiences for students with autism spectrum disorder: Personal identity, public disclosure, and institutional support. Journal of College Student Development, 58(1), 71-87.

Fernandes, M. C. M. D. O. Autismo, Estigma E Vulnerabilidade. http://www.fundamentalpsychopathology.org.br/wp-content/uploads/2020/07/MariaCristina-Maia-de-Oliveira-Fernandes_Autismo-estigma-e-vulnerabiliade_def_congressopsicopatologia-converted.pdf Acessado: 23 de fevereiro de 2021.

Gonçalves, E. C. M. (2020). Inclusão e permanência do estudante com transtorno do espectro autista na educação superior.

Mendonça Oliveira, A. F. T., \& de Abreu, T. F. (2019). A percepção do aluno com transtorno do espectro autista sobre o proces so de inclusão na Universidade Federal de Goiás (UFG). Revista Diálogos e Perspectivas em Educação Especial, 6(2), 59-76.

Olivati, A. G., \& Leite, L. P. (2019). Experiências acadêmicas de estudantes universitários com Transtornos do Espectro Autista: uma análise interpretativa dos relatos. Revista Brasileira de Educação Especial, 25(4), 729-746.

Ramos, C. S. A, Lemos, E. L. D. M. D., \& Salomão, N. M. R. (2019). Vivências Escolares e Transtorno do Espectro Autista: o que Dizem as Crianças? Rev. bras. educ. espec. vol, 25(3).

Sarrett, J. C. (2018). Autism and accommodations in higher education: Insights from the autism community. Journal of Autism and Developmental Disorders, 48(3), 679-693.

Silva, M. C., \& de Oliveira Pavão, S. M. (2018). Possibilidades das adaptações curriculares na educação superior. Revista e-Curriculum, 16(3), 621-649.

Silveira, P. T. D., Donida, L. O., \& Santana, A. P. (2020). Inclusão e permanência de universitários com diagnóstico de trans torno do espectro autista: discussões acerca de barreiras linguísticas. Avaliação: Revista da Avaliação da Educação Superior, 25(3), 659-675.

Souza, M. T. D., Silva, M. D. D., \& Carvalho, R. D. (2010). Revisão integrativa: o que é e como fazer. Einstein, 8(1), 102-106.

Wuo, A. S. (2019). Educação de pessoas com transtorno do espectro do autismo: estado do conhecimento em teses e dissertações nas regiões Sul e Sudeste do Brasil (2008-2016). Saúde e Sociedade, 28, 210-223. 